\title{
Sprachenpolitik und die Rolle der Fremdsprachen (Deutsch) in Südafrika
}

\author{
Carlotta von Maltzan \\ Department of Modern Foreign Languages, Stellenbosch University, Private Bag X1, Matieland 7602 \\ E-mail:vmaltzan@sun.ac.za
}

\begin{abstract}
Taking multilingualism in South Africa into account, this paper investigates language policy, language usage and the role of foreign languages, especially German, within the context of processes of transformation at universities since 1994, i.e. after the end of Apartheid. It is argued that foreign languages find themselves caught in the battle between ideology and practice and have to forge a new direction for themselves.
\end{abstract}

Keywords: language policy, South Africa, role of foreign languages, German, South African universities, education

Schlüsselbegriffe: Sprachenpolitik, Südafrika, Rolle der Fremdsprachen, Deutsch, Universitäten in Südafrika, Bildung

\section{Einleitung: Sprachenvielfalt in Südafrika}

In Südafrika hat sich nach 1994 die Bedeutung der Sprachen und insbesondere der Fremdsprachen an Schulen und Hochschulen mit der Transformation von einem Apartheidstaat in eine demokratische Gesellschaftsordnung entscheidend verändert. Zurückzuführen ist dieser Wandel auf ein neues Selbstverständnis, denn als wirtschaftliche Führungsmacht auf dem afrikanischen Kontinent und als multikulturelle Gesellschaft stellte sich Südafrika nicht nur der Herausforderung eine Neustrukturierung des gesamten Bildungssektors vorzunehmen, sondern in diesem Zusammenhang auch eine neue Sprachenpolitik zu entwickeln. Diese Entscheidung beruhte auf der Einsicht, dass in Südafrika zwar insgesamt etwa 25 Sprachen in nennenswertem Umfang gesprochen werden und als Muttersprachen der überwiegenden Mehrheit der Bevölkerung gelten können (Beukes 2004), wovon aber nur zwei, nämlich Englisch und Afrikaans, im öffentlichen Leben sowie an Schulen und Hochschulen eine Anwendung fanden und damit Vormachtstellung hatten. Vielsprachigkeit - und damit Mehrsprachigkeit - ist bekanntlich der Normalzustand in allen afrikanischen Ländern und wird es zunehmend in Ländern anderer Kontinente. Anders jedoch als in vielen afrikanischen Staaten, die nach der Unabhängigkeit manchmal neben einer einheimischen Sprache, oft aber nur die jeweilige Kolonialsprache zur Amtssprache erklärten, entschied man sich in Südafrika dafür elf Sprachen den Status einer Amtssprache zu verleihen. So kommt es, dass diese tatsächlich die jeweiligen Muttersprachen von $99 \%$ der Bevölkerung abdecken. Damit wurde der 1994 unter der Führung von Nelson Mandela 
entstandenen Vorstellung einer kulturellen Verständigung im Sinne der Versöhnung und gegenseitigen Toleranz im Zeichen der Regenbogennation genüge geleistet, da keiner Sprache und damit Bevölkerungsgruppe eine Vorrangstellung zugesprochen wurde. Die Muttersprachen der großen Mehrheit der ca. 44.8 Millionen Einwohner in Südafrika sind zwar afrikanischen Ursprungs, wobei jedoch auch hier keine Sprache von einer überwiegenden Mehrheit gesprochen wird, obgleich immerhin knapp über $40 \%$ der Bevölkerung entweder mit isiZulu (23.8\%) oder isiXhosa (17.6\%) als Muttersprache aufgewachsen sind (Beukes 2004). Es folgen der Anzahl der Sprecher nach geordnet Afrikaans mit 13\% sowie Sepedi, Englisch, Setswana und Sesotho, die alle von mehr als jeweils 8\% der Bevölkerung gesprochen werden, während die restlichen vier Landessprachen von nur jeweils weniger als 5\% der Bevölkerung gesprochen werden. Die afrikanischen Sprachen lassen sich dabei größtenteils in zwei Sprachgruppen, nämlich die Nguni- und Sotho-Sprachen unterteilen. Von den elf Landessprachen sind nur zwei europäischen Ursprungs, nämlich Englisch und Afrikaans, obgleich man sicherlich argumentieren könnte, wie z.B. Robert Kriger (1996) es tut, dass Afrikaans auch als afrikanische bzw. nicht-europäische Sprache gelten muss, da dreiviertel der sog. „Coloured“-Bevölkerung zur afrikaanssprachigen Gruppe gehört. Eine Reihe von europäischen Sprachen wie Portugiesisch, Griechisch, Italienisch und Deutsch werden von Minderheiten gesprochen, die statistisch gesehen aber unter 1\% bleiben. Größere Minderheiten haben indische Sprachen wie Hindi, Urdu, Gujarati und Tamil als Muttersprache und durch verstärkte Einwanderung aus anderen, vor allem Ländern des afrikanischen Kontinents ist auch die französischsprachige Minderheit gewachsen (Balladon 2006; Beukes 2004; Kriger 1996).

\section{Sprachenpolitik: Mehrsprachigkeit und Minderheitssprachen}

Auffällig ist allemal, dass in der südafrikanischen Verfassung von 1996 darauf Wert gelegt wurde keiner einzigen Sprache den Vorzug zu geben und etwa eine Sprache zu einer übergreifenden Landes- bzw. Nationalsprache zu deklarieren. Alle elf Sprachen sollten als gleichberechtigt oder zumindest als gleichwertig gelten. Bemerkenswert und vielleicht sogar ungewöhnlich ist in der südafrikanischen Sprachenpolitik darüber hinaus auch, dass eine Anzahl weiterer Sprachen in der Verfassung genannt werden, die zwar nicht zu den Nationalsprachen gezählt, aber durchaus als förderungswürdig angesehen und von Minderheiten, nämlich Nachkommen von Einwanderern oder von in den zwei vergangenen Jahrhunderten nach Südafrika verschleppten Arbeitskräften gesprochen werden. Explizit genannt werden in der Verfassung (Chapter 1, §6) Deutsch, Griechisch, Gujarati, Hindi, Portugiesisch, Tamil, Telegu und Urdu. Auch Arabisch, Hebräisch und Sanskrit werden erwähnt, die in den verschiedenen Religionsgemeinschaften Südafrikas eine wichtige Rolle einnehmen. „Die genannten Sprachen, darunter Deutsch, sind nur beispielhaft aufgeführt und stehen für ein offenes Kontinuum, in dem nach der Verfassung prinzipiell jede Sprache, die in Südafrika gesprochen wird, den Status einer schützenswerten südafrikanischen Sprache genießen kann." (Laurien 2006:440). Es gibt also, wie Laurien (ebd.) ganz richtig feststellt, per definitionem keine einheimischen Sprachen und keine Fremdsprachen, sondern eher mehr oder weniger häufig gesprochene Sprachen. Mit dieser Forderung nach einem weit reichenden Sprachenpluralismus gesteht die Verfassung zwar jeder Bevölkerungsgruppe in Südafrika ohne Rücksicht auf ihren Status das Recht auf eine eigene Sprache zu und verpflichtet sich gleichzeitig zur aktiven Förderung derjenigen Sprachen, die in der Apartheidzeit marginalisiert und ausgegrenzt worden waren, wohl um deren Status zu heben und deren Gebrauch zu unterstützen. Tatsächlich hat die Praxis mittlerweile gezeigt, dass heute nur Englisch eine Vorrangstellung hat und die übrigen der elf südafrikanischen Sprachen zwar in 
einem weitaus geringeren Umfang staatlich gefördert werden, die Sprachen von Nachkommen der Einwanderer jedoch kaum mehr, wie weiter unten besprochen wird.

Um oben genannte Verfassungsziele umzusetzen und eine aktive Sprachenpolitik zu fördern, wurde 1996 und mit einem weiteren Erlass von 1999 eine eigene Institution gegründet, nämlich das Pan South African Language Board (PanSALB). Dieses Gremium hat sich in Bezug auf Gesetzgebung, Sprachenpolitik und Sprachgebrauch eine ganze Reihe von Aufgaben gestellt, die in den Jahresberichten 2005/2006 sowie 2006/2007 nachzulesen sind und in einer sich mit Trommelschlag öffnenden Webseite unter dem Motto „One Nation, many Languages" folgendermaßen vorgestellt werden:

(a) make recommendations with regard to any proposed or existing legislation, practice and policy dealing directly or indirectly with language matters at any level of government, and with regard to any proposed amendments to or the repeal or replacement of such legislation, practice and policy;

(b) make recommendations to organs of state at all levels of government where it considers such action advisable for the adoption of measures aimed at the promotion of multilingualism within the framework of the Constitution;

(c) advise on the co-ordination of language planning in South Africa.

(Quelle: http://www.pansalb.org.za/pansalbhistory.html)

Ziel der südafrikanischen Sprachenpolitik ist also nicht die Durchsetzung des gleichzeitigen Gebrauchs aller elf Amtssprachen auf allen Ebenen im öffentlichen Raum, sondern vielmehr die Anerkennung einer mehrdimensionalen Mehrsprachigkeit an sich. Die Herausforderung in Südafrika ist daher, unter Berücksichtung regionaler Unterschiede sowie der jeweiligen geographischen und institutionellen Situation, den Sprachengebrauch auf die gleichzeitige Benutzung von zwei oder drei Sprachen auszuhandeln. De facto sind die meisten Südafrikaner ohnehin mehrsprachig und beherrschen neben ihrer Muttersprache immer noch eine bis zwei Zweitsprachen, wobei weiße Südafrikaner in den seltensten Fällen eine der afrikanischen Sprachen beherrschen, während schwarze Südafrikaner meist neben ihrer Muttersprache und Englisch oder Afrikaans mindestens auch eine der beiden großen afrikanischen Sprachen wie IsiXosa oder isiZulu und anderer Sprachen mächtig sind. Obgleich also Mehrsprachigkeit in allen Bereichen dezidiert gefördert wird, können sich die meisten Sprachen und insbesondere diejenigen von Minderheiten nur durch den eigentlichen Gebrauch erhalten, da sie in einem schulischen und universitären Kontext und damit in der Bildung meist keine Bedeutung haben oder höchstens einen sekundären Rang einnehmen, indem schon allein wegen der Verständigungsschwierigkeiten Minderheitssprachen zwar außerhalb des Unterrichts, aber nicht im Klassenzimmer oder in Hörsälen erwünscht sind. Eine Ausnahme bildet in diesem Zusammenhang die südafrikanische Gebärdensprache, die mittlerweile - will man sich an die Auflistung des Pan South African Language Board halten - als inoffizielle zwölfte Amtssprache anerkannt worden ist, was heißen will, dass es inzwischen üblich ist z.B. Nachrichtensendungen auch mit Gebärdensprache zu übertragen.

\section{Sprachgebrauch und Bildung: Zwischen Ideologie und Praxis}

Der Sprachgebrauch im Bereich der Bildung und Ausbildung an Schulen und Hochschulen war und ist ein heiß umstrittenes Thema. Schon allein die Tatsache, dass sich der Aufstand von 1976 in Soweto an der umstrittenen Einführung von Afrikaans als Unterrichtssprache in Schulen der Townships entzündete und schließlich die Proteste, Streiks und Demonstrationen der 1980er Jahre und die politischen Umwälzungen der 1990er Jahre einleiteten, zeigt bereits 
die Brisanz dieses Themas. Dafür gibt es historische Gründe, wie z.B. Verordnungen, nach denen an Schulen immer schon die europäischen Sprachen wie zunächst Holländisch (und seit 1925 Afrikaans) oder Englisch eine Vorrangstellung hatten und damit die Muttersprachen der weißen Minderheitsbevölkerung privilegierte. Erst der sog. Bantu Education Act von 1953 verlieh den Muttersprachen afrikanischen Ursprungs einen wenn auch begrenzten Status, da er vorsah, dass schwarze Schüler zumindest in den Primarstufen Anspruch auf Unterricht in ihrer Muttersprache hatten. Dieses scheinbar durchaus sinnvolle Anliegen wirkte sich allerdings insofern nachteilig aus, als eine ohnehin schon eingeschränkte Bildung unter dem Apartheidregime in der Muttersprache nur begrenzt ausgeführt werden konnte, da diese zum Teil noch nicht vollständig verschriftlicht waren noch das erforderliche Lehrmaterial in diesen Sprachen zur Verfügung stand. Diesem historisch und politisch begründeten Defizit in der von der Apartheidregierung gesteuerten Bildungspolitik versuchte ein dem Parlament vorgelegter Erlass des Erziehungsministeriums durch den National Education Policy Act (Act 27) von 1996 entgegenzutreten, der 1997 in eine Verabschiedung der Language-in-Education Policy mündete. Diese zweite Verordnung ist vor allem auch insofern von Bedeutung, als sie entscheidend auf das Angebot von Fremdsprachen und damit auch des Deutschen an südafrikanischen Schulen Einfluss genommen und $\mathrm{zu}$ einem starken Rückgang von Fremdsprachen an Hochschulen geführt hat.

Im Wesentlichen beruht laut Paragraph 1 die neue Verordnung auf einem Paradigma, das kulturelle Vielfalt im neuen Nationalstaat in deutlicher Absetzung zur Vergangenheit in der Formel der Regenbogennation als durchweg positiv bewertet. Kulturelle Unterschiede werden deshalb ebenso wie Mehrsprachigkeit begrüßt. Man geht dabei von der Erkenntnis aus, dass die im Bildungssektor vorherrschende Sprachenpolitik der Vergangenheit politisch motiviert war und zu rassistisch begründeter und sprachlicher Diskriminierung führte, die die Zulassung und den Zugang ins Bildungssystem und den Erfolg vieler Lerner entscheidend beeinträchtigte. Deshalb versteht sich die im neuen Südafrika eingeführte Sprachenpolitik explizit als Teil einer Strategie der Regierung, den Aufbau eines nicht-rassischen (non-racist) Nationalstaates jenseits der Grenzen von Hautfarbe, Sprache und Religion zum Zwecke gegenseitiger Verständigung zu fördern. Das Erlernen von mehr als einer Sprache gehört mithin zum Ausgangspunkt und Mehrsprachigkeit zum Selbstverständnis jedes Südafrikaners, um ethnisch begründetem Chauvinismus von vorneherein die Spitze abzubrechen. Allerdings schreibt das Erziehungsministerium nicht direkt vor welche Sprachen an der Schule eingeführt oder in welcher Sprache unterrichtet werden muss. Ob der Unterricht in einer Muttersprache abgehalten und durch das Erlernen von einer weiteren oder mehreren Sprachen ergänzt oder aber auf dem Modell eines bilingualen Unterrichts aufgebaut werden sollte, bleibt zunächst der jeweiligen Schulbehörde überlassen, so lang das grundlegende Prinzip der Förderung von Mehrsprachigkeit im Unterricht gefördert wird.

Grundsätzlich gelten im Ausbildungsbereich laut Paragraph 5 des gleichen Gesetzes folgende Regelungen hinsichtlich des Sprachgebrauchs:

1. Eltern haben das Recht für ihre Kinder zu wählen, in welcher Sprache sie unterrichtet werden.

2. Wenn Unterricht in einer bestimmten Sprache nicht angeboten wird, kann ein entsprechender Antrag auf Einführung dieser Sprache bei der Schulbehörde gestellt werden. 
3. Jede Schule muss Mehrsprachigkeit fördern, entweder durch bilingualen Unterricht oder Unterricht in einer Sprache mit mindestens zwei anderen Sprachen als Pflichtfächern.

(Quelle: salanguages.com)

Das 2005 neu eingeführte Curriculum für Schulen fördert Mehrsprachigkeit, indem es zunächst die Muttersprachen der Schüler und erst dann andere Sprachen und Kulturen fördert. Damit werden Schüler scheinbar dazu befähigt, ,informed choices“ zu treffen bei der Entscheidung sich weitere Sprachen anzueignen. Diese Funktionalisierung der Sprachen je nach Lebensbedingungen und Regionen ist praktisch gesehen nur schwer durchsetzbar, solange eine massive Ungleichheit der Sprachen in der gesellschaftlichen Bewertung bestehen bleibt (Laurien 2006:441). De facto ist es nämlich so, dass trotz aller gegenläufigen Gesetzgebungen und Bemühungen Mehrsprachigkeit in Südafrika durchzusetzen und diese auch im Bildungssektor zu verankern, Englisch als Umgangs- und Verkehrssprache sich immer stärker durchsetzt und Staatssprache, die Sprache der Politik, der Justiz und der Medien ist. Das hängt damit zusammen, dass Englisch als prestigeträchtige Sprache, also als Sprache der Macht und des finanziellen und professionellen Erfolgs wahrgenommen wird (Balladon 2006:54). Wie schon verschiedentlich festgestellt (Alexander 2001; Beukes 2004; Balladon 2006) ist es trotz guten Willens bei einer nur schleppenden Umsetzung von Vielsprachigkeit geblieben, die sich zudem in einer sehr widersprüchlichen Bildungspolitik äußert. An diesem Tatbestand hat sich auch in den letzten Jahren nichts geändert.

Dennoch ist an der südafrikanischen Verfassung und an der Bildungspolitik bemerkenswert, dass der Umgang mit Mehrsprachigkeit und unterschiedlichen kulturellen Gruppen und Interessen nicht auf der Betonung von Unterschieden beharrt, sondern vielmehr gegenseitigen Respekt und gegenseitige Toleranz fordert. Der Kulturbegriff ist daher ein dynamischer, der nicht auf der Festlegung von Differenzen besteht, sondern vielmehr den Bildungsprozess als einen dialogischen Austausch von Wertesystemen ansieht, wie es der ehemalige Erziehungsminister Kader Asmal (2002:4) folgendermaßen zum Ausdruck bringt „Values cannot be simply asserted. They must be placed on the table, debated, negotiated, synthesised, modified and earned. And this process, this dialogue, is in itself a value - a South African value - to be cherished." In der Verhandlung von Werten und ihrer Anerkennung entsteht also eine Bildung, deren Bedingung eine Erziehung zu transkultureller Kompetenz ist. Dass dieser Prozess bei weitem nicht abgeschlossen, zum Teil mühsam und nicht immer unproblematisch ist, weil er in einen viel größeren Transformationsprozess eingebettet ist, in dem es gilt die Kluft zwischen Arm und Reich, zwischen Rasse und Klasse zu überwinden, haben u.a. die gewalttätigen und xenophobischen Ausschreitungen gegenüber Migranten, vor allem aus anderen Ländern Afrikas zwischen Mai und Juli 2008 gezeigt.

\section{Zur Rolle der Fremdsprachen, insbesondere des Deutschen}

Vor diesem Hintergrund ist vor allem ein Tatbestand frappierend, nämlich dass in Südafrika europäische Sprachen wie z.B. Portugiesisch, Deutsch, Französisch, Italienisch und Griechisch sowie auch die schon genannten indischen Sprachen zwar der Einstufung nach nicht als Fremdsprachen sondern vielmehr als (zwar privilegierte) Minderheitensprachen gelten, sie aber trotz der gleichwertigen Verankerung in der Gesetzgebung und der von der Regierung deklarierten Verpflichtung zur Mehrsprachigkeit und der Beteuerung alle Sprachen im Bildungsbereich zu fördern, zunehmend ausgegrenzt werden. Mehrsprachigkeit an den Schulen zum Beispiel wird nämlich nur bedingt gefördert. Jeder Schüler muss zwei 
Amtssprachen erlernen, nämlich die Muttersprache und eine zusätzliche Sprache. Obgleich es grundsätzlich möglich ist, an Schulen auch eine dritte Sprache zu belegen, ist das Curriculum so eng gefasst, dass das Erlernen einer dritten Sprache den Lerner mit Sicht auf langfristige Ausbildungsziele und derzeitiger Zulassungsbedingungen zur Universität erheblich einschränkt. Hinzu kommt, dass vor allem Fremdsprachen vom Erziehungsministerium eher als unwichtig erachtet werden, da es nicht einmal ausgefeilte, geschweige denn gedruckte Lehrpläne für Fremdsprachen gibt (Balladon 2006:53). Theorie und Praxis klaffen also hinsichtlich der Sprachenpolitik und ihrer Umsetzung in Südafrika weit auseinander.

Im Kontext dieser Kluft haben in den letzten Jahren Fremdsprachen an sich und auch jene, die in der Verfassung explizit genannt werden, wegen mangelnder Unterstützung und der vom Erziehungsministerium vorgegebenen finanziellen Einschränkungen einen entscheidenden Bedeutungsverlust erlitten und zu einem Rückgang im Angebot dieser Fächer an Schulen und Universitäten geführt, so auch für das Fach Deutsch. Die Einführung des National Curriculum 2005 war nicht nur für das Fach Deutsch eine Herausforderung, denn Stellenstreichungen an Schulen und Hochschulen, Einsparungen und mangelnder Nachwuchs verlangen eine kreative Auseinandersetzung mit dem gesellschaftlichen Umfeld von Lernern und Studierenden. Lehrinhalte und Lernziele mussten im Zuge der Qualitätssicherung im Sinne einer outcomes based education neu bestimmt werden. Neue Prüfungsbestimmungen, ein allgemein gehaltener Lehrplan für Deutsch (nur auf der Webseite des Erziehungsministeriums, aber nicht in Druckfassung erhältlich), mangelnde oder unzureichende Kommunikation zwischen Erziehungsministerium, Prüfungsbehörden und Schulen, Unklarheiten über Zuständigkeitsbereiche, kurzfristige Planung führen bei Lehrern und Fachberatern zu Unsicherheit und sogar zu der pessimistischen Einschätzung, dass „das Ende des Weges erreicht sei“ (Rode 2008:27). Vor allem an afrikaanssprachigen Schulen und Universitäten war die deutsche Sprache vor 1994 wegen der Nähe zu Afrikaans verbreitet und auch heute befinden sich die anteilmäßig meisten Deutschlerner dort. Insgesamt ist die Anzahl der Deutschlerner in den letzten 20 Jahren erheblich geschrumpft, so dass derzeit an rund 110 südafrikanischen Schulen mit Hilfe von 120 Lehrern ca. 13000 Schüler unterrichtet werden ${ }^{1}$. Außerdem bietet das Goethe-Institut Johannesburg Sprachkurse an, ein von der Bundesrepublik Deutschland entsandter Fachberater für Deutsch als Fremdsprache kümmert sich um die Fortbildung und Förderung von Deutschlehrern in der nördlichen Region, während in der südlichen Kapprovinz noch ein Fachberater für Fremdsprachen, darunter Deutsch, vom dortigen Erziehungsministerium angestellt ist, der Lehrer von Fremdsprachen an allen Schulen berät, wobei jedoch Deutsch vornehmlich an den afrikaanssprachigen Schulen angeboten wird.

An Universitäten ist die Lage für das Hochschulfach Deutsch ähnlich komplex und hat sich inhaltlich und strukturell in den letzten 20 Jahren erheblich verändert. Bis in die 1970er Jahre wurde an allen „weißen“ sowie an 4 ,nichtweißen“ Universitäten vor allem Literaturwissenschaft mit unterschiedlicher Schwerpunktsetzung im Rahmen eines dreijährigen B.A.-Studiengangs unterrichtet. Umstrukturierungen ab Mitte der 1980er Jahre hingen z. T. mit Neubesetzungen von Lehrstühlen zusammen oder entstanden aufgrund von Rationalisierungsmaßnahmen (Kußler 2001). Im Laufe der Jahre wurden alle eigenständigen Seminare meist mit anderen europäischen Sprachen (z.B. Französisch oder Italienisch) zunächst in Departments, später in noch größere Einheiten, meist „Schools“ zusammengelegt. Dennoch ergibt sich ein überraschendes Bild hinsichtlich der Anzahl der Deutschstudierenden über den Zeitraum 1994 bis 2008 (Tabelle 1). Interessant ist nämlich, dass die Gesamtanzahl der Deutschstudierenden im Bachelorstudiengang nicht nur zwischen 1994 und 2003 mit ca. 
1200 Studierenden landesweit gleich geblieben ist (Annas 2004:190), sondern auch, dass 2008 insgesamt 1195 Studierende das Fach Deutsch an Universitäten belegen. Prozentmäßig verteilt sich die Anzahl der Studierenden wie folgt: im 1. Studienjahr sind es 70.4\%, im 2. Studienjahr 22\% und im 3. Studienjahr nur 7.6\%. Das bedeutet, dass anteilmäßig mehr als zwei Drittel aller Studierenden nur ein Jahr lang Deutsch als Fach belegen. Allerdings ist der Umstand gleich bleibender Studierendenzahlen in den letzten 15 Jahren von Bedeutung, denn im gleichen Zeitraum wurden nicht nur Deutschabteilungen an drei von 16 Universitäten, wo das Fach Deutsch angeboten wurde, geschlossen, sondern gleichzeitig fand auch ein starker Stellenabbau an allen Universitäten statt. So sank die Anzahl der Planstellen für das Fach Deutsch von 56 im Jahr 1994 auf insgesamt 25 im Jahr 2008. Wie sich aus Tabelle 1 ablesen lässt, ist allerdings auch bemerkenswert, dass an vier Universitäten (Stellenbosch, Rhodes, Free State und North West) ein deutlicher Zuwachs an Studierenden zu verzeichnen ist, während an den übrigen Universitäten die Zahlen teils minimal, in vielen Fällen sogar erheblich geschrumpft sind, so dass 2009 eine weitere Deutschabteilung (Pietermaritzburg) geschlossen wurde. Wenn sich dieser allgemeine Trend auch in den nächsten 15 Jahren bestätigen sollte, kann man davon ausgehen, dass in der Zukunft zwar noch weitere Deutschabteilungen von einer möglichen Schließung bedroht sind, jedoch die Präsenz des Fachs an den meisten Hochschulen in Südafrika nicht gefährdet scheint. Deutsch als Fremdsprache und germanistische Arbeit an sich befindet sich schon seit etlichen Jahren und auch jetzt noch an vielen Universitäten auf Grund der oft geringen Studierendenzahlen in einer Legitimationskrise und wird dennoch, sicher auch auf Grund der südafrikanischen Sprachenpolitik und dem Bewusstsein einer facettenreichen Mehrsprachigkeit ausgesetzt zu sein, trotz allgemeiner Sparmaßnahmen gehalten. Durch neue Programmentwürfe sowie Partnerschaften mit deutschen Universitäten und gemeinsam entwickelten Studiengängen sowie durch verstärkte Zusammenarbeit mit Deutschabteilungen in anderen afrikanischen Ländern kann dieser Krise m. E. durchaus konstruktiv begegnet werden. Immerhin sind landesweit zwischen 2003 und 2008 die Zahlen der eingeschriebenen Studierenden in Postgraduate Studiengängen um fast ein Drittel, nämlich von 22 auf 31 angestiegen ${ }^{3}$. Insofern ist die derzeitige Entwicklung an den Universitäten für das Fach Deutsch im deutlichen Gegensatz zu der Entwicklung an den Schulen durchaus positiv zu bewerten, insofern nämlich, als sich das Fach neben anderen Fremdsprachen und trotz der teils widersprüchlichen Sprachenpolitik durchaus und gerade in einem mehrsprachigen Kontext, wenn auch in verringertem Maße und an weniger Universitäten behaupten kann. 
Tabelle 1. Studierende im Fach Deutsch (Undergraduate/ Bachelor Degree) 1994 und $2008^{2}$

\begin{tabular}{|c|c|c|c|c|c|c|c|c|c|c|}
\hline Universitäit & 1994 & & & & & 2008 & & & & \\
\hline & Anf. & D1 & D2 & D3 & Ges. & Anf. & D1 & D2 & D3 & Ges. \\
\hline University of Cape Town & 40 & & 10 & 10 & 60 & 15 & & 10 & 10 & 35 \\
\hline $\begin{array}{l}\text { University of the Western } \\
\text { Cape }\end{array}$ & 33 & 4 & 12 & 7 & 56 & 27 & & 5 & 3 & 35 \\
\hline University of Stellenbosch & 135 & 43 & 13 & 13 & 204 & 156 & 32 & 111 & 23 & 322 \\
\hline $\begin{array}{l}\text { Nelson Mandela Metropolitan } \\
\text { University (University of Port } \\
\text { Elizabeth) }\end{array}$ & 15 & 6 & 8 & 3 & 32 & & & & & $\mathbf{0}$ \\
\hline Rhodes University & 52 & 5 & 10 & 1 & 68 & 54 & & 16 & 7 & 77 \\
\hline $\begin{array}{l}\text { Walter Sisulu University for } \\
\text { Technology and Science } \\
\text { (University of Transkei) }\end{array}$ & & & & & $\mathbf{0}$ & & & & & $\mathbf{0}$ \\
\hline University of the Free State & 11 & 13 & 13 & 7 & 44 & 30 & 12 & 8 & 6 & 56 \\
\hline $\begin{array}{l}\text { University of KwaZulu-Natal } \\
\text { (Durban) }\end{array}$ & & & & & 0 & 30 & & 10 & 2 & 42 \\
\hline $\begin{array}{l}\text { University of KwaZulu-Natal } \\
\text { (Pietermaritzburg) }\end{array}$ & 25 & 2 & 15 & 4 & 46 & & & 2 & 3 & 5 \\
\hline University of Zululand & 33 & 0 & 8 & 2 & 43 & 35 & & & 1 & 36 \\
\hline $\begin{array}{l}\text { North-West University } \\
\text { (Potchefstroom) }\end{array}$ & 25 & 3 & 0 & 0 & 28 & 152 & & 17 & 7 & 176 \\
\hline $\begin{array}{l}\text { University of the } \\
\text { Witwatersrand, Johannesburg }\end{array}$ & 55 & & 15 & 9 & 79 & 10 & & 9 & 8 & 27 \\
\hline $\begin{array}{l}\text { University of Johannesburg } \\
\text { (Randse Afrikaanse } \\
\text { Universiteit) }\end{array}$ & 20 & 10 & 14 & 6 & $\mathbf{5 0}$ & 21 & 6 & 7 & 1 & 35 \\
\hline University of Pretoria & 62 & 34 & 18 & 8 & 122 & 64 & 25 & 9 & 3 & 101 \\
\hline University of South Africa & 88 & 113 & 75 & 32 & 308 & 152 & & 53 & 14 & 219 \\
\hline $\begin{array}{l}\text { University of Limpopo } \\
\text { (University of the North) }\end{array}$ & & & & & 47 & 20 & 0 & 6 & 3 & 29 \\
\hline Gesamtzahlen & & & & & 1187 & & & & & 1195 \\
\hline
\end{tabular}

Aufschlüsselung: Anf. = Anfänger; Ges. = Gesamtzahlen; D1= Studierende mit Vorkenntnissen in Deutsch (Schulabschluss); D2, D3 = Studierende im zweiten bzw. dritten Studienjahr.

\section{Fazit und Ausblick}

Die Bildungslandschaft ist im Hinblick auf die Rolle, die den Fremdsprachen im Kontext der neuen Sprachenpolitik zugewiesen worden ist, unausgewogen und trotz begrüßenswerter Ansätze das allgemeine Bildungsniveau zu heben und allen Lernern gleiche Chancen zu bieten widersprüchlich. Zwar sind in der Verfassung Sprachenpluralismus und transkulturelle Kommunikation als Werte fest geschrieben, doch besonders an Schulen werden durch die Vorschrift, zwei dieser Sprachen als Fächer zu belegen insbesondere die Amtssprachen 
gefördert, wobei in der Praxis vor allem Englisch eine Vorrangstellung einnimmt. Diese Hervorhebung führt, wie Balladon (2006:63) das nennt, zu einem ,Safrozentrismus', d.h. zu einer Innenschau auf vorwiegend südafrikanische Verhältnisse. Die Vorstellung einer kulturellen Vielfalt und des Sprachenpluralismus, die als Modell für andere Länder gelten und somit Richtung weisend sein könnte, scheint mithin auf Südafrika zugeschnitten zu sein, nicht aber über die Landesgrenzen hinaus zu weisen. Fest steht jedoch auch, dass sich gerade die Fremdsprachen und damit auch das Fach Deutsch vor dem Hintergrund der Bildungsreformen der vergangenen Jahre und angesichts einer weit reichenden Bildungspolitik, die ein neues Selbstverständnis in einem mehrsprachigen Kontext kreiert hat, neu orientieren und verorten müssen, da sie trotz der derzeitigen institutionellen und finanziellen Einschränkungen durch eine aktive Auseinandersetzung mit der südafrikanischen Mehrsprachigkeit eine Öffnung nach außen bewirken können.

\section{Anmerkungen}

1. Diese Zahlen beruhen auf einer Statistik von 2009, die jährlich von Rudolf Rode, dem Fachberater für Fremdsprachen an der Erziehungsbehörde der Provinz Western Cape, erhoben wird und die er mir zur Verfügung gestellt hat.

2. Diese von mir durchgeführte Erhebung für Studierendenzahlen von 2008 knüpft an Ergebnisse von Rolf Annas (2004) an und beruht auf Angaben, die mir von allen Deutschabteilungen in Südafrika zur Verfügung gestellt wurden. Da im Laufe der letzten Jahre viele Universitäten umstrukturiert und auch umbenannt wurden, wird der 1994 geführte Name der jeweiligen Universität in der Tabelle in Klammern genannt. Die Walter Sisulu University for Technology and Science (früher University of Transkei) wurde trotz fehlender Zahlen aufgeführt, weil zwar für 1994 keine Zahlen zur Verfügung stehen, aber noch 2003 dort insgesamt 35 Studierende Deutsch belegt hatten (vgl. Annas 2004). Mittlerweile wurde auch diese Deutschabteilung geschlossen.

3. Die von mir durchgeführte Erhebung von 2008 für Studierendenzahlen in Postgraduate Studiengängen knüpft an die Ergebnisse und Zahlen der Erhebung aus dem Jahr 2003 von Rolf Annas (2004) an. 2003 waren landesweit für Honours 8 (2008 sind 11) eingeschrieben. 2003 gab es im Magister/Masterstudiengang 6 Studierende, 2008 waren es 9, während die Zahl der eingeschriebenen Studierenden für den $\mathrm{PhD}$ (Promotion) von 9 im Jahr 2003 auf 11 im Jahr 2008 anstieg.

\section{Bibliographie}

Alexander, N. and K. Heugh. 2001. Language Policy in the New South Africa. In R. Kriger and A. Zegeye (Hgg.) Culture in the New South Africa. After Apartheid-Volume two. Cape Town: Kwela Books and SA History Online. pp. 15-40

Annas, R. 2004. Zur Situation des Faches Deutsch an südafrikanischen Universitäten. Acta Germanica 30/31: 181-191.

Asmal, K. 2002. Pride versus Arrogance. In K. Asmal and W. James (Hgg.) Spirit of the Nation, Reflections on South Africa's Educational Ethos. Claremont: Human Sciences Research Council and the Department of Education. pp. 2-10.

Balladon, F. 2006. The challenge of diversity: The National Curriculum Statement and foreign languages. Journal for Language Teaching 40(2): 49-66.

Beukes, A.M. 2004. The first ten years of democracy: Language Policy in South Africa. Paper read at the World Congress on Linguistic Diversity, Sustainability and Peace, Barcelona 20-23 May 2004. Online verfügbar unter http://lapica-unesco.cesca.es/congres04/pdf/1_ beukes.pdf; letzter Zugriff am 14.08. 2009. 
Kußler, R. 2001. Deutschunterricht und Germanistikstudium in Südafrika. In G. Helbig, L. Götze, G. Henrici und H.J. Krumm (Hgg.) Deutsch als Fremdsprache. Ein internationales Handbuch. 2. Halbband. Berlin / New York: De Gruyter. pp. 1609-1619.

Kriger, R. 1996. Afrikaans: Recollection, Redefinition, Restitution. Introduction. In R. Kriger und E. Kriger (Hgg.) Afrikaans Literature. Recollection, Redefinition, Restitution. Papers held at the 7th congress on South African Literature at the Protestant Academy Bad Boll. Amsterdam, Atlanta: Rodopi. pp. 9-12

Laurien, I. 2006. Das Fach Deutsch an Universitäten im „Neuen Südafrika“ - Eine „Laborsituation“ für Europa? Info DaF 33(5): 438-445.

Pan South African Language Board (PanSALB): One Nation, many languages. Online verfügbar unter http://www.pansalb.org.za/index.html; letzter Zugriff am 14.08. 2009.

Pan South African Language Board: Annual Report 2005/2006. Online verfügbar unter http://www.pansalb.org.za/2005-2006\%20Annual\%20Report.pdf; letzter Zugriff am 12.08. 2009.

Pan South African Language Board: Annual Report 2006/2007. Fostering Multilingualism beyond the 21st Century. Online verfügbar unter http://www.pansalb.org.za/ PanSALB\%20AR\% 202007\%20(2).pdf; letzter Zugriff am 12.08. 2009.

Rode, R. 2008. Deutsch an südafrikanischen Schulen. Eine Bestandsaufnahme. eDUSA 3(2): 26-29.

\section{Biographical note}

Carlotta von Maltzan is Professor of German and Chair of Modern Foreign Languages at the University of Stellenbosch. She published books on Heiner Müller and Klaus Mann as well as various articles. She also edited two volumes on German/African literature. Her current research focuses on writing about Africa in German fiction. She is editor of Acta Germanica. 